\title{
HIGHER-ORDER SPACE-TIME CONTINUOUS GALERKIN METHODS FOR THE WAVE EQUATION
}

\author{
Marco Zank ${ }^{1}$ \\ ${ }^{1}$ Faculty of Mathematics \\ University of Vienna \\ Oskar-Morgenstern-Platz 1, 1090 Wien, Austria \\ marco.zank@univie.ac.at
}

Key words: Wave Equation, Space-Time, Continuous Galerkin, Finite Elements, Higher-Order, CFL Condition

\begin{abstract}
We consider a space-time variational formulation of the second-order wave equation, where integration by parts is also applied with respect to the time variable. Conforming tensor-product finite element discretisations with piecewise polynomials of this space-time variational formulation require a CFL condition to ensure stability. To overcome this restriction in the case of piecewise multilinear, continuous ansatz and test functions, a stabilisation is well-known, which leads to an unconditionally stable space-time finite element method. In this work, we generalise this stabilisation idea from the lowestorder case to the higher-order case, i.e. to an arbitrary polynomial degree. We give numerical examples for a one-dimensional spatial domain, where the unconditional stability and optimal convergence rates in space-time norms are illustrated.
\end{abstract}

\section{INTRODUCTION}

Standard approaches for the numerical solution of hyperbolic initial-boundary value problems are usually based on semi-discretisations in space and time, where the discretisation in space and time is split accordingly. In contrast to these approaches, space-time methods discretise time-dependent partial differential equations without separating the temporal and spatial directions. In this work, the homogeneous Dirichlet problem for the second-order wave equation,

$$
\left.\begin{array}{rlrl}
\partial_{t t} u(x, t)-\Delta_{x} u(x, t) & =f(x, t) & & \text { for }(x, t) \in Q=\Omega \times(0, T), \\
u(x, t) & =0 & & \text { for }(x, t) \in \Sigma=\partial \Omega \times[0, T], \\
u(x, 0)=\partial_{t} u(x, 0) & =0 & & \text { for } x \in \Omega,
\end{array}\right\}
$$

serves as a model problem, where $\Omega=(0, L)$ is an interval for $d=1$, or $\Omega$ is polygonal for $d=2$, or $\Omega$ is polyhedral for $d=3, T>0$ is a terminal time and $f$ is a given right-hand side. To derive a space-time variational formulation, we define the space-time Sobolev spaces

$$
\begin{aligned}
& H_{0 ; 0}^{1,1}(Q):=L^{2}\left(0, T ; H_{0}^{1}(\Omega)\right) \cap H_{0,}^{1}\left(0, T ; L^{2}(\Omega)\right) \subset H^{1}(Q), \\
& H_{0 ;, 0}^{1,1}(Q):=L^{2}\left(0, T ; H_{0}^{1}(\Omega)\right) \cap H_{, 0}^{1}\left(0, T ; L^{2}(\Omega)\right) \subset H^{1}(Q)
\end{aligned}
$$


with the Hilbertian norms

$$
\|v\|_{H_{0 ; 0,0}^{1,1}(Q)}:=\|v\|_{H_{0 ; 0}^{1,1}(Q)}:=|v|_{H^{1}(Q)}:=\left(\int_{0}^{T} \int_{\Omega}\left(\left|\partial_{t} v(x, t)\right|^{2}+\left|\nabla_{x} v(x, t)\right|^{2}\right) \mathrm{d} x \mathrm{~d} t\right)^{1 / 2},
$$

where $v \in H_{0,}^{1}\left(0, T ; L^{2}(\Omega)\right)$ satisfies $\|v(\cdot, 0)\|_{L^{2}(\Omega)}=0$ and $w \in H_{, 0}^{1}\left(0, T ; L^{2}(\Omega)\right)$ fulfils $\|w(\cdot, T)\|_{L^{2}(\Omega)}=0$, see [5] for more details. The bilinear form

$$
a(\cdot, \cdot): H_{0 ; 0,}^{1,1}(Q) \times H_{0 ;, 0}^{1,1}(Q) \rightarrow \mathbb{R},
$$

defined by the variational identity

$$
a(u, w):=-\left\langle\partial_{t} u, \partial_{t} w\right\rangle_{L^{2}(Q)}+\left\langle\nabla_{x} u, \nabla_{x} w\right\rangle_{L^{2}(Q)}
$$

for $u \in H_{0 ; 0,}^{1,1}(Q)$ and $w \in H_{0 ;, 0}^{1,1}(Q)$, is continuous, i.e. the estimate

$$
\forall u \in H_{0 ; 0,1}^{1,1}(Q): \forall w \in H_{0 ;, 0}^{1,1}(Q): \quad|a(u, w)| \leq|u|_{H^{1}(Q)}|w|_{H^{1}(Q)}
$$

holds true due to the Cauchy-Schwarz inequality. The space-time variational formulation of (1) is to find $u \in H_{0 ; 0}^{1,1}(Q)$ such that

$$
\forall w \in H_{0 ;, 0}^{1,1}(Q): \quad a(u, w)=\langle f, w\rangle_{L^{2}(Q)},
$$

where $f \in L^{2}(Q)$ is a given right-hand side. Note that the initial condition $u(\cdot, 0)=0$ is considered in the strong sense, whereas the existence and uniquenes Theorem 1.1 For $f \in$ and the stability estimate
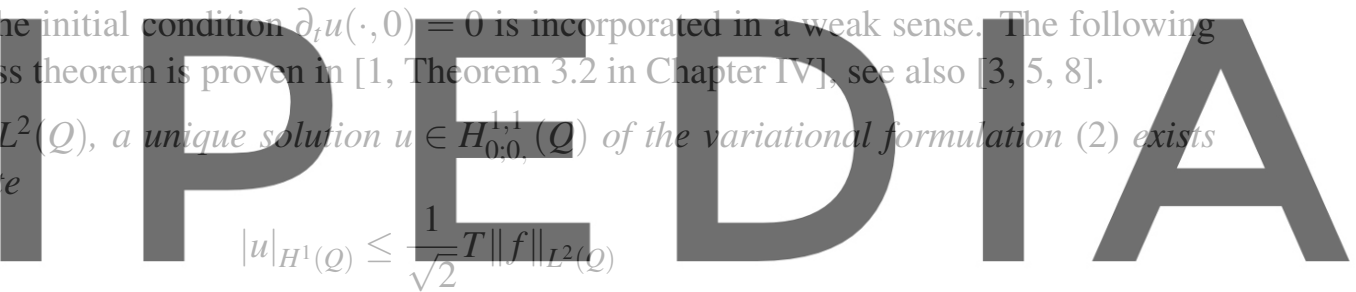

Register for free at https//www.scipedia.com to download the version without the watermark Note that the solution operator

$$
\mathcal{L}: L^{2}(Q) \rightarrow H_{0 ; 0,}^{1,1}(Q), \quad \mathcal{L} f:=u,
$$

of Theorem 1.1 is not an isomorphism, i.e. $\mathcal{L}$ is not surjective, see $[4,5]$ for more details. In this work, for simplicity, we only consider homogeneous initial conditions, where inhomogeneous initial conditions can be treated analogously as in $[1,7,8]$.

A conforming tensor-product space-time discretisation of (2) with piecewise polynomial, continuous ansatz and test functions requires a CFL condition

$$
h_{t} \leq C h_{x}
$$

with a constant $C>0$, depending on the constant of a spatial inverse inequality, where $h_{t}$ and $h_{x}$ are the mesh sizes in time and space. For a one-dimensional spatial domain $\Omega$, i.e. $d=1$, and piecewise multilinear, continuous ansatz and test functions, the CFL condition (3) reads as

$$
h_{t}<h_{x}
$$


for uniform meshes with uniform mesh sizes $h_{t}$ and $h_{x}$, see [3, 5]. To overcome the CFL condition (3), the stabilised space-time finite element method to find $u_{h} \in\left(V_{h_{x}, 0}^{1}(\Omega) \otimes S_{h_{t}}^{1}(0, T)\right) \cap H_{0 ; 0}^{1,1}(Q)$ such that

$$
-\left\langle\partial_{t} u_{h}, \partial_{t} w_{h}\right\rangle_{L^{2}(Q)}+\sum_{\alpha=1}^{d}\left\langle\partial_{x_{\alpha}} u_{h}, Q_{h_{t}}^{0} \partial_{x_{\alpha}} w_{h}\right\rangle_{L^{2}(Q)}=\left\langle f, w_{h}\right\rangle_{L^{2}(Q)}
$$

for all $w_{h} \in\left(V_{h_{x}, 0}^{1}(\Omega) \otimes S_{h_{t}}^{1}(0, T)\right) \cap H_{0 ;, 0}^{1,1}(Q)$ was analysed in $[2,5,8]$, where

$$
Q_{h_{t}}^{0}: L^{2}(Q) \rightarrow L^{2}(\Omega) \otimes S_{h_{t}}^{0}(0, T)
$$

is the extended $L^{2}$ projection on the space of the temporal piecewise constant functions and $V_{h_{x}, 0}^{1}(\Omega) \otimes$ $S_{h_{t}}^{1}(0, T)$ is the space of piecewise multilinear, continuous functions, see Section 2 for the notations. The main results for this proposed space-time finite element method (4) are the unconditional stability, i.e. no CFL condition is needed, and the space-time error estimates with

$$
h:=\max \left\{h_{x}, h_{t}\right\}, \quad h_{x}=\max _{k} h_{x, k}, \quad h_{t}=\max _{\ell} h_{t, \ell}
$$

which are summarised in the following theorem, where its proof is contained in [2, 5].

Theorem 1.2 There exists a unique solution $u_{h} \in\left(V_{h_{x}, 0}^{1}(\Omega) \otimes S_{h_{t}}^{1}(0, T)\right) \cap H_{0 ; 0}^{1,1}(Q)$ of $(4)$, satisfying the $L^{2}(Q)$ stability estimate
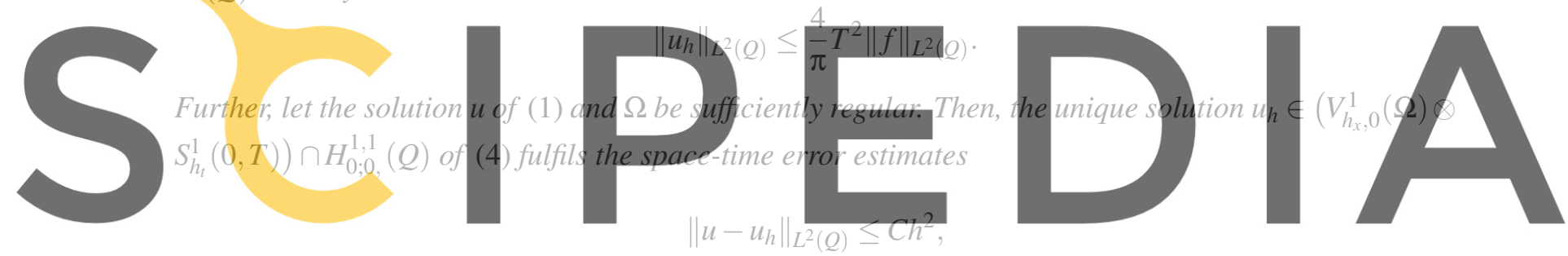

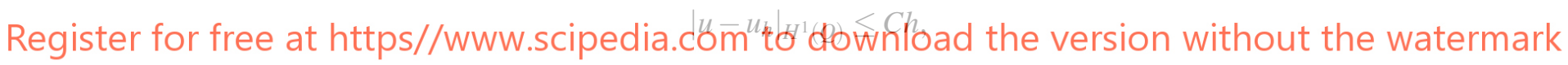

where, for the $H^{1}(Q)$ error estimate, a spatial inverse inequality is additionally assumed.

In this work, we generalise this stabilisation idea from the linear case to the higher-order case. In greater detail, we introduce a new stabilised space-time finite element method of tensor-product type with globally continuous ansatz and test functions, which are piecewise polynomials of an arbitrary polynomial degree $p$, leading to unconditional stability and optimal convergence rates in the space-time norms $\|\cdot\|_{L^{2}(Q)}$, $|\cdot|_{H^{1}(Q)}$. In other words, the result of Theorem 1.2 is generalised to an arbitrary polynomial degree $p$. The rest of the paper is organised as follows: In Section 2, notations of the used finite element spaces and $L^{2}$ projections are fixed. Section 3 introduces the new space-time finite element method. Numerical examples for a one-dimensional spatial domain and piecewise polynomials of higher-order are presented in Section 4. Finally, we draw some conclusions in Section 5.

\section{PRELIMINARIES}

In this section, notations of the used finite element spaces and $L^{2}$ projections are stated. For this purpose, let the bounded Lipschitz domain $\Omega \subset \mathbb{R}^{d}$ be an interval $\Omega=(0, L)$ for $d=1$, or polygonal for $d=2$, or 
polyhedral for $d=3$. For a tensor-product ansatz, we consider admissible decompositions

$$
\bar{Q}=\bar{\Omega} \times[0, T]=\bigcup_{k=1}^{N_{x}} \overline{\omega_{k}} \times \bigcup_{\ell=1}^{N_{t}}\left[t_{\ell-1}, t_{\ell}\right]
$$

with $N:=N_{x} \cdot N_{t}$ space-time elements, where the time intervals $\tau_{\ell}:=\left(t_{\ell-1}, t_{\ell}\right)$ with mesh sizes $h_{t, \ell}=$ $t_{\ell}-t_{\ell-1}$ are defined via the decomposition

$$
0=t_{0}<t_{1}<t_{2}<\cdots<t_{N_{t}-1}<t_{N_{t}}=T
$$

of the time interval $(0, T)$. The maximal and the minimal time mesh sizes are denoted by $h_{t}:=h_{t, \max }:=$ $\max _{\ell} h_{t, \ell}$ and $h_{t, \min }:=\min _{\ell} h_{t, \ell}$, respectively. For the spatial domain $\Omega$, we consider a shape-regular sequence $\left(\mathcal{T}_{v}\right)_{v \in \mathbb{N}}$ of admissible decompositions

$$
\mathcal{T}_{v}:=\left\{\omega_{k} \subset \mathbb{R}^{d}: k=1, \ldots, N_{x}\right\}
$$

of $\Omega$ into finite elements $\omega_{k} \subset \mathbb{R}^{d}$ with mesh sizes $h_{x, k}$, the maximal mesh size $h_{x}:=h_{x, \max }:=\max _{k} h_{x, k}$ and the minimal mesh size $h_{x, \min }:=\min _{k} h_{x, k}$. The spatial elements $\omega_{k}$ are intervals for $d=1$, triangles or quadrilaterals for $d=2$, and tetrahedra or hexahedra for $d=3$. Next, for a fixed polynomial degree $p \in \mathbb{N}$, we introduce the finite element space

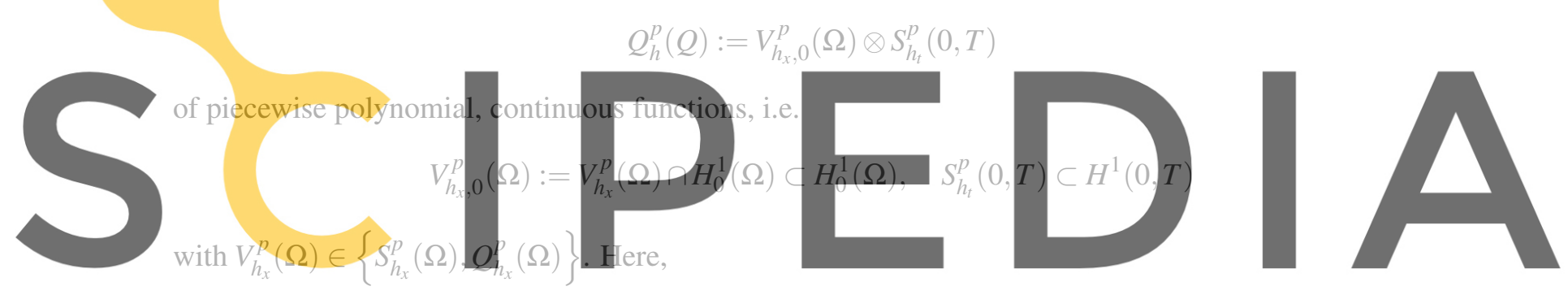

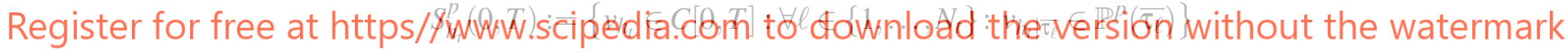

denotes the space of piecewise polynomial, continuous functions on intervals, where $\mathbb{P}^{p}(A)$ is the space of polynomials on a subset $A \subset \mathbb{R}^{d}$ of global degree at most $p$. Analogously,

$$
S_{h_{x}}^{p}(\Omega):=\left\{v_{h_{x}} \in C(\bar{\Omega}): \forall \omega \in \mathcal{T}_{v}: v_{h_{x} \mid \bar{\omega}} \in \mathbb{P}^{p}(\bar{\omega})\right\}
$$

is the space of piecewise polynomial, continuous functions on intervals $(d=1)$, triangles $(d=2)$, or tetrahedra $(d=3)$. Moreover,

$$
Q_{h_{x}}^{p}(\Omega):=\left\{v_{h_{x}} \in C(\bar{\Omega}): \forall \omega \in \mathcal{T}_{v}: v_{h_{x} \mid \bar{\omega}} \in \mathbb{Q}^{p}(\bar{\omega})\right\}
$$

is the space of piecewise polynomial, continuous functions on intervals $(d=1)$, quadrilaterals $(d=2)$, or hexahedra $(d=3)$, where $\mathbb{Q}^{p}(A)$ is the space of polynomials on a subset $A \subset \mathbb{R}^{d}$ of degree at most $p$ in each variable. The temporal nodal basis functions of $S_{h_{t}}^{p}(0, T)$ are denoted by $\varphi_{n}^{p}$ for $n=0, \ldots, p N_{t}$, and $\psi_{j}^{p}, j=1, \ldots, M_{x}$, are the spatial nodal basis functions of $V_{h_{x}, 0}^{p}(\Omega)$, i.e.

$$
S_{h_{t}}^{p}(0, T)=\operatorname{span}\left\{\varphi_{n}^{p}\right\}_{n=0}^{p N_{t}} \quad \text { and } \quad V_{h_{x}, 0}^{p}(\Omega)=\operatorname{span}\left\{\psi_{j}^{p}\right\}_{j=1}^{M_{x}} .
$$


For the stabilisation of the new space-time finite element method, we also need the spaces of piecewise polynomial, discontinuous functions

$$
S_{h_{t}}^{q, \text { disc }}(0, T):=\left\{v_{h_{t}} \in L^{1}(0, T): \forall \ell \in\left\{1, \ldots, N_{t}\right\}: v_{h_{t} \mid \tau_{\ell}} \in \mathbb{P}^{q}\left(\tau_{\ell}\right)\right\},
$$

where $q \in \mathbb{N}_{0}$ is a fixed polynomial degree. For a given function $v \in L^{2}(Q)$, the extended $L^{2}$ projection $Q_{h_{t}}^{q \text {,disc }} v \in L^{2}(\Omega) \otimes S_{h_{t}}^{q \text {,disc }}(0, T)$ on the space $L^{2}(\Omega) \otimes S_{h_{t}}^{q \text {, disc }}(0, T)$ of piecewise polynomial, discontinuous functions with respect to the time variable is defined by

$$
\left\langle Q_{h_{t}}^{q \text {,disc }} v, v_{h_{t}}\right\rangle_{L^{2}(Q)}=\left\langle v, v_{h_{t}}\right\rangle_{L^{2}(Q)}
$$

for all $v_{h_{t}} \in L^{2}(\Omega) \otimes S_{h_{t}}^{q \text {, disc }}(0, T)$, satisfying the stability estimate

$$
\left\|Q_{h_{t}}^{q, \text { disc }} v\right\|_{L^{2}(Q)} \leq\|v\|_{L^{2}(Q)} .
$$

Note that $Q_{h^{\prime}}^{0}=Q_{h}^{0, \text { disc }}$ is the extended $L^{2}$ projection (5) on the space of the temporal piecewise constant functions $L^{2}(\Omega) \otimes S_{h_{t}}^{0}(0, T)=L^{2}(\Omega) \otimes S_{h_{t}}^{0, \text { disc }}(0, T)$. Analogously, for a solely time-dependent function $w \in L^{2}(0, T)$, we denote $Q_{h_{t}}^{q, \text { disc }} w \in S_{h_{t}}^{q \text {,disc }}(0, T)$ as the $L^{2}(0, T)$ projection on the space $S_{h_{t}}^{q \text {,disc }}(0, T)$ of piecewise polynomial, discontinuous functions, defined by

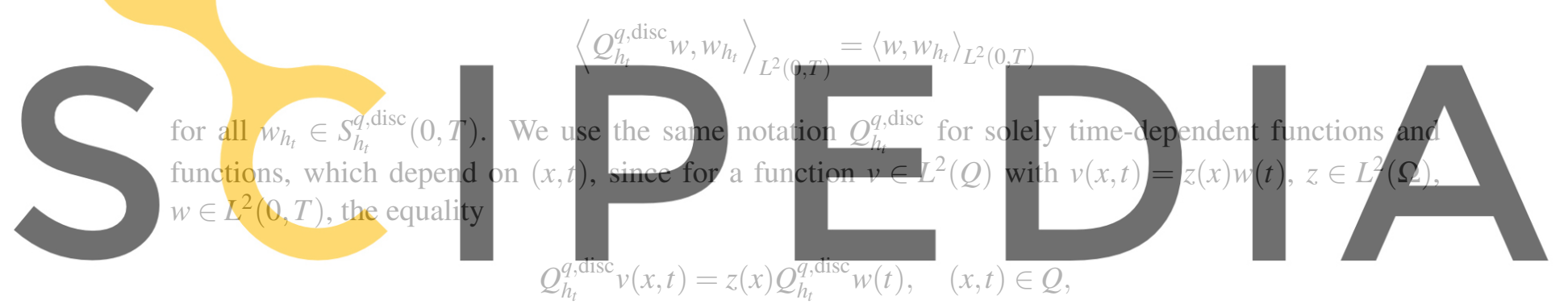

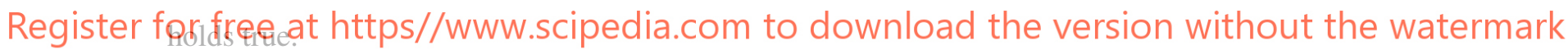

\section{NEW STABILISED SPACE-TIME FINITE ELEMENT METHOD}

In this section, we introduce a new stabilised space-time finite element method with continuous ansatz and test functions, which are piecewise polynomials of arbitrary polynomial degree $p \in \mathbb{N}$ with respect to the spatial variable and the temporal variable. For this purpose, we fix a polynomial degree $p \in \mathbb{N}$ and we introduce the perturbed bilinear form

$$
a_{h}(\cdot, \cdot): Q_{h}^{p}(Q) \cap H_{0 ; 0}^{1,1}(Q) \times Q_{h}^{p}(Q) \cap H_{0 ;, 0}^{1,1}(Q) \rightarrow \mathbb{R}
$$

by defining

$$
a_{h}\left(u_{h}, w_{h}\right):=-\left\langle\partial_{t} u_{h}, \partial_{t} w_{h}\right\rangle_{L^{2}(Q)}+\sum_{\alpha=1}^{d}\left\langle\partial_{x_{\alpha}} u_{h}, Q_{h_{t}}^{p-1, \text { disc }} \partial_{x_{\alpha}} w_{h}\right\rangle_{L^{2}(Q)}
$$

for $u_{h} \in Q_{h}^{p}(Q) \cap H_{0 ; 0,}^{1,1}(Q), w_{h} \in Q_{h}^{p}(Q) \cap H_{0 ; 0}^{1,1}(Q)$. Note that the function $\partial_{x_{\alpha}} w_{h}, \alpha=1, \ldots, d$, fulfils

$$
\partial_{x_{\alpha}} w_{h} \in L^{2}(\Omega) \otimes S_{h_{t}}^{p}(0, T),
$$


i.e. $\partial_{x_{\alpha}} w_{h}$ is still a piecewise polynomial of degree $p$ with respect to the temporal variable. The perturbed bilinear form $a_{h}(\cdot, \cdot)$ is continuous since the Cauchy-Schwarz inequality and the $L^{2}(Q)$ stability (6) of $Q_{h_{t}}^{p-1, \text { disc }}$ yield

$$
\left|a_{h}\left(u_{h}, w_{h}\right)\right| \leq\left|u_{h}\right|_{H^{1}(Q)}\left|w_{h}\right|_{H^{1}(Q)}
$$

for all $u_{h} \in Q_{h}^{p}(Q) \cap H_{0 ; 0,1}^{1,1}(Q), w_{h} \in Q_{h}^{p}(Q) \cap H_{0 ; 0}^{1,1}(Q)$. The perturbed variational formulation, corresponding to (2), is to find $u_{h} \in Q_{h}^{p}(Q) \cap H_{0 ; 0}^{1,1}(Q)$ such that

$$
\forall w_{h} \in Q_{h}^{p}(Q) \cap H_{0 ;, 0}^{1,1}(Q): a_{h}\left(u_{h}, w_{h}\right)=\left\langle f, w_{h}\right\rangle_{L^{2}(Q)} .
$$

This perturbed variational formulation (7) coincides with the perturbed variational formulation (4) for $p=1$. In other words, the new perturbed variational formulation (7) is a generalisation of the perturbed variational formulation (4) from $p=1$ to arbitrary $p \in \mathbb{N}$. The numerical analysis, i.e. an analogous result as Theorem 1.2, of the perturbed variational formulation (7) is far beyond the scope of this contribution, we refer to [6].

The discrete variational formulation (7) is equivalent to the linear system

$$
K_{h} \underline{u}=\underline{f}
$$

with the system matrix

$$
K_{h}:=-A_{h_{t}} \otimes M_{h_{x}}+\widetilde{M}_{h_{t}} \otimes A_{h_{x}} \in \mathbb{R}^{M_{x} \cdot p N_{t} \times M_{x} \cdot p N_{t}},
$$

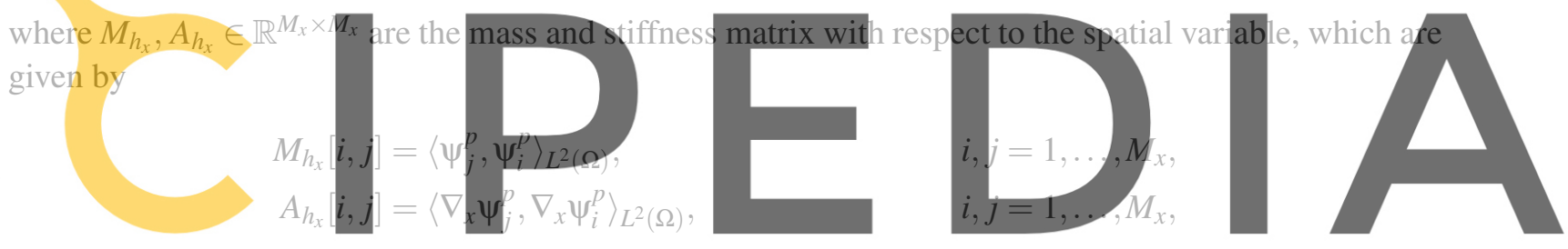

and $\widetilde{M}_{h_{t}}, A_{h_{t}} \in \mathbb{R}^{p N_{t} \times p N_{t}}$ are the perturbed mass and stiffness matrix with respect to temporal variable,

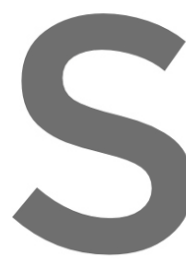

Register forfree att https//www.scipedia.com to download the version without the watermark

$$
\begin{aligned}
\widetilde{M}_{h_{t}}[n, m] & =\left\langle\varphi_{m}^{p}, Q_{h_{t}}^{p-1, \text { disc }} \varphi_{n}^{p}\right\rangle_{L^{2}(0, T)}, & & n=0, \ldots, p N_{t}-1, m=1, \ldots, p N_{t}, \\
A_{h_{t}}[n, m] & =\left\langle\partial_{t} \varphi_{m}^{p}, \partial_{t} \varphi_{n}^{p}\right\rangle_{L^{2}(0, T)}, & & n=0, \ldots, p N_{t}-1, m=1, \ldots, p N_{t} .
\end{aligned}
$$

Here, the nodal basis function $\varphi_{0}^{p}$ corresponds to the vertex $t_{0}=0$ and the nodal basis function $\varphi_{p N_{t}}^{p}$ corresponds to the vertex $t_{N_{t}}=T$. As the $L^{2}(0, T)$ projection $Q_{h_{t}}^{p-1 \text {,disc }}$ can be computed locally, i.e. on each temporal element $\tau_{\ell}$ for $\ell=1, \ldots, N_{t}$, the assembling of the perturbed mass matrix $\widetilde{M}_{h_{t}}$ can be realised, as for the classical mass matrix, via local matrices.

\section{NUMERICAL EXAMPLES}

In this section, numerical examples for the new space-time finite element method (7) are given. For this purpose, we consider the hyperbolic initial-boundary value problem (1) in the one-dimensional spatial domain $\Omega:=(0,1)$ with the terminal time $T=10$, i.e. the rectangular space-time domain

$$
Q:=\Omega \times(0, T):=(0,1) \times(0,10) .
$$


As exact solutions, we choose

$$
\begin{aligned}
& u_{1}(x, t)=t^{2} \sin (10 \pi x) \sin (t x), \\
& u_{2}(x, t)=t^{2}(T-t)^{9 / 5} \sqrt{t+x^{2}+1} \sin (\pi x)
\end{aligned}
$$

for $(x, t) \in Q$. The spatial domain $\Omega=(0,1)$ is decomposed into nonuniform elements with the vertices

$$
x_{0}=0, \quad x_{1}=1 / 4, \quad x_{2}=1,
$$

whereas the temporal domain $(0, T)=(0,10)$ is decomposed into nonuniform elements with the vertices

$$
t_{0}=0, \quad t_{1}=T / 8, \quad t_{2}=T / 4, \quad t_{3}=T .
$$

We apply a uniform refinement strategy for the meshes (12), (13), which do not fulfil the CFL condition (3) at least for piecewise multilinear, continuous functions, i.e. $p=1$. Additionally, we choose $p=1$ for Table 1, $p=2$ for Table 2, and $p=6$ for Table 3, where the number of the degrees of freedom is denoted by

$$
\operatorname{dof}=M_{x} \cdot p \cdot N_{t} .
$$

The global linear system (8) is solved by a direct solver, where the appearing integrals to compute the related right-hand side are calculated by using high-order quadrature rules.

In the case of piecewise multilinear, continuous functions, i.e. $p=1$, the numerical results for the smooth

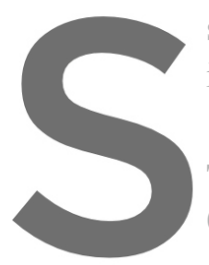

solution $u_{1}$ in (10) are givent

in $\|\cdot\|_{L^{2}(Q)}$ and Yinear con

Table 1: Numerical result (9) for the smooth function
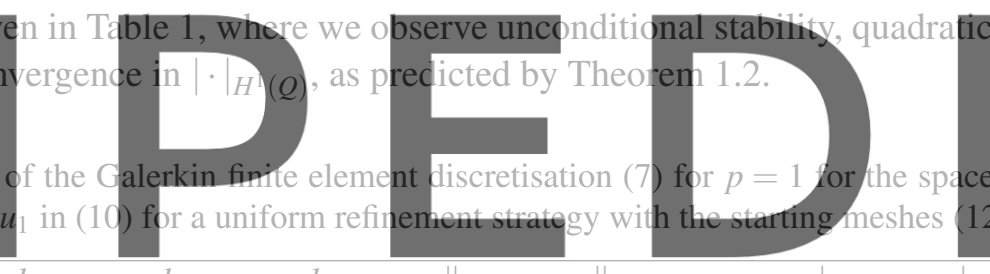

dof $h_{x, \max }$

$h_{x, \min }$

$h_{t, \max }$

$h_{t, \min }$

eoc

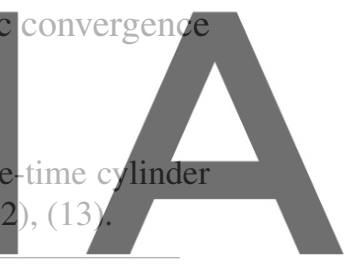

eoc

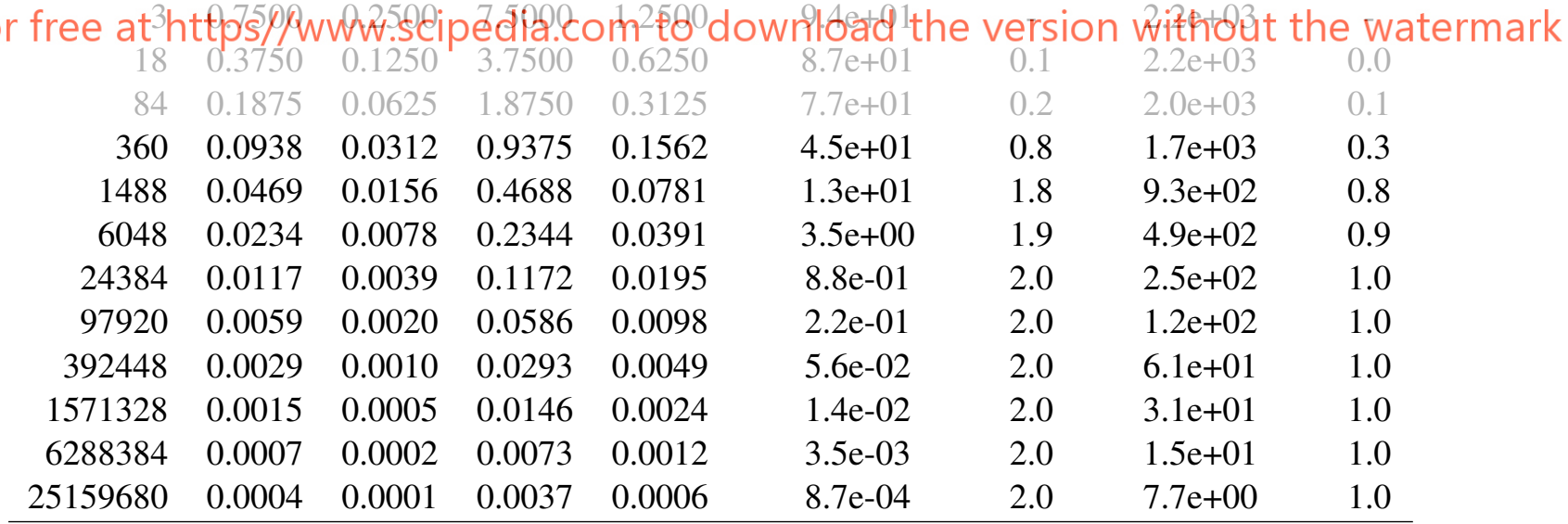

For $p=2$ and $p=6$, the results for the smooth solution $u_{1}$ in (10) are stated in Table 2 and Table 3, respectively, where we illustrate that the new space-time finite element method (7) is unconditionally stable and the convergence rates with respect to the space-time norms $\|\cdot\|_{L^{2}(Q)},|\cdot|_{H^{1}(Q)}$ are as expected. Moreover, a comparison of Table 1, Table 2 and Table 3 show that a polynomial degree $p>1$ is advisable 
since the numbers of the degrees of freedom are much lower for $p>1$ than for $p=1$ when a fixed accuracy is desired. For example, we need dof $=25159680$ degrees of freedom for $p=1$, dof $=392448$ degrees of freedom for $p=2$ and dof $=13680$ degrees of freedom for $p=6$ to receive the error in $|\cdot|_{H^{1}(Q)}$ within a comparable range.

Table 2: Numerical results of the Galerkin finite element discretisation (7) for $p=2$ for the space-time cylinder (9) for the smooth function $u_{1}$ in (10) for a uniform refinement strategy with the starting meshes (12), (13).

\begin{tabular}{rcccccccc}
\hline dof & $h_{x, \max }$ & $h_{x, \min }$ & $h_{t, \max }$ & $h_{t, \min }$ & $\left\|u_{1}-u_{1, h}\right\|_{L^{2}(Q)}$ & eoc & $\left|u_{1}-u_{1, h}\right|_{H^{1}(Q)}$ & eoc \\
\hline 18 & 0.7500 & 0.2500 & 7.5000 & 1.2500 & $4.4 \mathrm{e}+03$ & - & $1.4 \mathrm{e}+04$ & - \\
84 & 0.3750 & 0.1250 & 3.7500 & 0.6250 & $7.8 \mathrm{e}+01$ & 5.8 & $2.1 \mathrm{e}+03$ & 2.8 \\
360 & 0.1875 & 0.0625 & 1.8750 & 0.3125 & $4.6 \mathrm{e}+01$ & 0.8 & $1.7 \mathrm{e}+03$ & 0.3 \\
1488 & 0.0938 & 0.0312 & 0.9375 & 0.1562 & $1.2 \mathrm{e}+01$ & 2.0 & $7.5 \mathrm{e}+02$ & 1.2 \\
6048 & 0.0469 & 0.0156 & 0.4688 & 0.0781 & $2.6 \mathrm{e}+00$ & 2.2 & $2.4 \mathrm{e}+02$ & 1.7 \\
24384 & 0.0234 & 0.0078 & 0.2344 & 0.0391 & $2.2 \mathrm{e}-01$ & 3.6 & $5.7 \mathrm{e}+01$ & 2.1 \\
97920 & 0.0117 & 0.0039 & 0.1172 & 0.0195 & $2.6 \mathrm{e}-02$ & 3.1 & $1.4 \mathrm{e}+01$ & 2.0 \\
392448 & 0.0059 & 0.0020 & 0.0586 & 0.0098 & $3.2 \mathrm{e}-03$ & 3.0 & $3.6 \mathrm{e}+00$ & 2.0 \\
1571328 & 0.0029 & 0.0010 & 0.0293 & 0.0049 & $4.0 \mathrm{e}-04$ & 3.0 & $9.0 \mathrm{e}-01$ & 2.0 \\
6288384 & 0.0015 & 0.0005 & 0.0146 & 0.0024 & $5.1 \mathrm{e}-05$ & 3.0 & $2.2 \mathrm{e}-01$ & 2.0 \\
25159680 & 0.0007 & 0.0002 & 0.0073 & 0.0012 & $6.3 \mathrm{e}-06$ & 3.0 & $5.6 \mathrm{e}-02$ & 2.0 \\
\hline
\end{tabular}
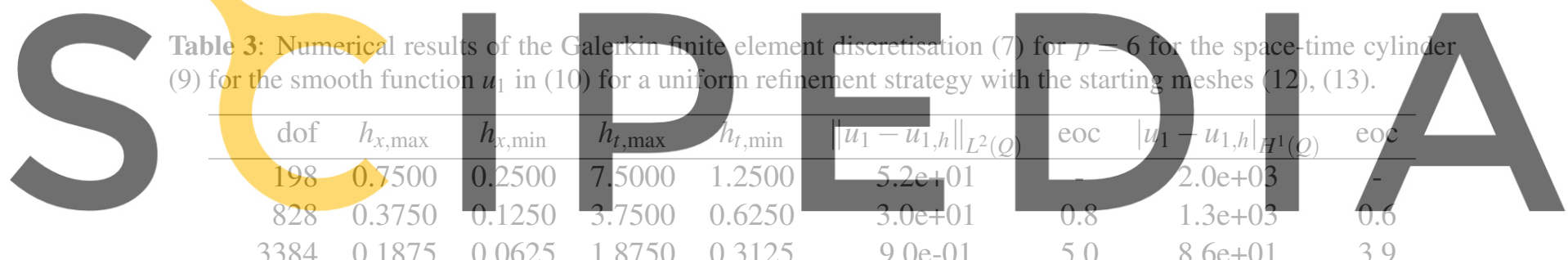

Register for

33840.1875

$\begin{array}{lll}0.0625 & 1.8750 & 0.3125\end{array}$

9.0e-01

$8.6 \mathrm{e}+01$

3.9

\begin{tabular}{|c|c|c|c|c|c|c|c|c|}
\hline 55008 & 0.0469 & 0.0156 & 0.4688 & 0.0781 & 8.0e-05 & 6.8 & $3.1 \mathrm{e}-02$ & 5.8 \\
\hline 220608 & 0.0234 & 0.0078 & 0.2344 & 0.0391 & $6.4 e-07$ & 7.0 & 4.9e-04 & 6.0 \\
\hline 883584 & 0.0117 & 0.0039 & 0.1172 & 0.0195 & $5.0 \mathrm{e}-09$ & 7.0 & $7.7 \mathrm{e}-06$ & 6.0 \\
\hline
\end{tabular}

For the singular solution $u_{2}$ in (11), the related results are given in Table 4 for $p=1$, Table 5 for $p=2$ and Table 6 for $p=6$, where we observe for $p>1$ a reduced order of convergence in $\|\cdot\|_{L^{2}(Q)}$ and in $|\cdot|_{H^{1}(Q)}$. These convergence rates correspond to the reduced Sobolev regularity $u_{2} \in H^{23 / 10-\varepsilon}(Q), \varepsilon>0$.

\section{CONCLUSIONS}

In this work, we introduced new stabilised higher-order space-time continuous Galerkin methods for the wave equation with globally continuous ansatz and test functions, which are piecewise polynomials of arbitrary polynomial degree. These methods are based on a space-time variational formulation, using also integration by parts with respect to the time variable, and its discretisation of tensor-product type with the help of a certain stabilisation. Thus, we generalised the well-known stabilisation idea from the lowest-order case to the higher-order case, i.e. to an arbitrary polynomial degree. We gave numerical 
Table 4: Numerical results of the Galerkin finite element discretisation (7) for $p=1$ for the space-time cylinder (9) for the singular function $u_{2}$ in (11) for a uniform refinement strategy with the starting meshes (12), (13).

\begin{tabular}{rcccccccc}
\hline dof & $h_{x, \max }$ & $h_{x, \min }$ & $h_{t, \max }$ & $h_{t, \min }$ & $\left\|u_{2}-u_{2, h}\right\|_{L^{2}(Q)}$ & eoc & $\left|u_{2}-u_{2, h}\right|_{H^{1}(Q)}$ & eoc \\
\hline 3 & 0.7500 & 0.2500 & 7.5000 & 1.2500 & $1.1 \mathrm{e}+03$ & - & $4.4 \mathrm{e}+03$ & - \\
18 & 0.3750 & 0.1250 & 3.7500 & 0.6250 & $7.2 \mathrm{e}+02$ & 0.6 & $2.9 \mathrm{e}+03$ & 0.6 \\
84 & 0.1875 & 0.0625 & 1.8750 & 0.3125 & $3.1 \mathrm{e}+02$ & 1.2 & $1.4 \mathrm{e}+03$ & 1.0 \\
360 & 0.0938 & 0.0312 & 0.9375 & 0.1562 & $8.7 \mathrm{e}+01$ & 1.8 & $5.6 \mathrm{e}+02$ & 1.4 \\
1488 & 0.0469 & 0.0156 & 0.4688 & 0.0781 & $2.4 \mathrm{e}+01$ & 1.9 & $2.5 \mathrm{e}+02$ & 1.2 \\
6048 & 0.0234 & 0.0078 & 0.2344 & 0.0391 & $6.5 \mathrm{e}+00$ & 1.9 & $1.1 \mathrm{e}+02$ & 1.1 \\
24384 & 0.0117 & 0.0039 & 0.1172 & 0.0195 & $1.6 \mathrm{e}+00$ & 2.0 & $5.6 \mathrm{e}+01$ & 1.0 \\
97920 & 0.0059 & 0.0020 & 0.0586 & 0.0098 & $4.1 \mathrm{e}-01$ & 2.0 & $2.8 \mathrm{e}+01$ & 1.0 \\
392448 & 0.0029 & 0.0010 & 0.0293 & 0.0049 & $1.0 \mathrm{e}-01$ & 2.0 & $1.4 \mathrm{e}+01$ & 1.0 \\
1571328 & 0.0015 & 0.0005 & 0.0146 & 0.0024 & $2.6 \mathrm{e}-02$ & 2.0 & $7.0 \mathrm{e}+00$ & 1.0 \\
6288384 & 0.0007 & 0.0002 & 0.0073 & 0.0012 & $6.5 \mathrm{e}-03$ & 2.0 & $3.5 \mathrm{e}+00$ & 1.0 \\
25159680 & 0.0004 & 0.0001 & 0.0037 & 0.0006 & $1.6 \mathrm{e}-03$ & 2.0 & $1.7 \mathrm{e}+00$ & 1.0 \\
\hline
\end{tabular}

Tabie 5: Numerical results of the Galerkin finite element discretisation (7) for $p=2$ for the space-time cylinder (9) for the singular function $u_{2}$ in (11) for a uniform refinement strategy with the starting meshes (12), (13).
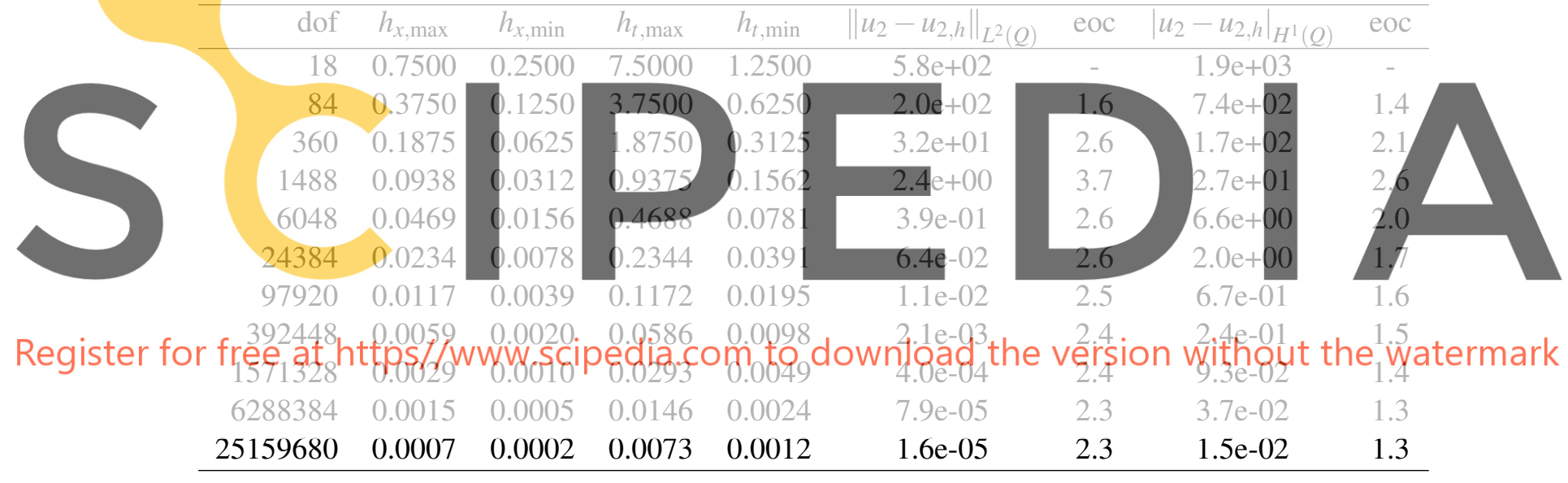

Table 6: Numerical results of the Galerkin finite element discretisation (7) for $p=6$ for the space-time cylinder (9) for the singular function $u_{2}$ in (11) for a uniform refinement strategy with the starting meshes (12), (13).

\begin{tabular}{rcccccccc}
\hline dof & $h_{x, \max }$ & $h_{x, \min }$ & $h_{t, \max }$ & $h_{t, \min }$ & $\left\|u_{2}-u_{2, h}\right\|_{L^{2}(Q)}$ & eoc & $\left|u_{2}-u_{2, h}\right|_{H^{1}(Q)}$ & eoc \\
\hline 198 & 0.7500 & 0.2500 & 7.5000 & 1.2500 & $2.7 \mathrm{e}+00$ & - & $1.6 \mathrm{e}+01$ & - \\
828 & 0.3750 & 0.1250 & 3.7500 & 0.6250 & $6.2 \mathrm{e}-01$ & 2.1 & $3.5 \mathrm{e}+00$ & 2.2 \\
3384 & 0.1875 & 0.0625 & 1.8750 & 0.3125 & $8.2 \mathrm{e}-02$ & 2.9 & $8.8 \mathrm{e}-01$ & 2.0 \\
13680 & 0.0938 & 0.0312 & 0.9375 & 0.1562 & $1.5 \mathrm{e}-02$ & 2.4 & $3.3 \mathrm{e}-01$ & 1.4 \\
55008 & 0.0469 & 0.0156 & 0.4688 & 0.0781 & $3.0 \mathrm{e}-03$ & 2.3 & $1.3 \mathrm{e}-01$ & 1.3 \\
220608 & 0.0234 & 0.0078 & 0.2344 & 0.0391 & $6.1 \mathrm{e}-04$ & 2.3 & $5.3 \mathrm{e}-02$ & 1.3 \\
883584 & 0.0117 & 0.0039 & 0.1172 & 0.0195 & $1.2 \mathrm{e}-04$ & 2.3 & $2.1 \mathrm{e}-02$ & 1.3 \\
\hline
\end{tabular}


examples, where the unconditional stability, i.e. no CFL condition is required, and optimal convergence rates in space-time norms were illustrated.

\section{REFERENCES}

[1] LADYZHENSKAYA, O. A. The boundary value problems of mathematical physics, vol. 49 of Applied Mathematical Sciences. Springer-Verlag, New York, 1985.

[2] Steinbach, O., AND ZANK, M. A stabilized space-time finite element method for the wave equation. In Advanced Finite Element Methods with Applications. Selected papers from the 30th Chemnitz FEM Symposium 2017, (T. Apel, U. Langer, A. Meyer, O. Steinbach eds.), Lecture Notes in Computational Science and Engineering. Springer, 2019, pp. 315-342.

[3] Steinbach, O., And Zank, M. Coercive space-time finite element methods for initial boundary value problems. Electron. Trans. Numer. Anal. 52 (2020), 154-194.

[4] Steinbach, O., And ZAnK, M. A generalized inf-sup stable variational formulation for the wave equation. [math.NA] 2101.06293, arXiv.org, 2021.

[5] ZANK, M. Inf-sup stable space-time methods for time-dependent partial differential equations. volume 36 of Monographic Series TU Graz: Computation in Engineering and Science. Feb 2020.

[6] ZANK, M. Stability and error estimates in space-time norms of a stabilized space-time finite element method for the wave equation for an arbitrary polynomial degree. In preparation (2021).

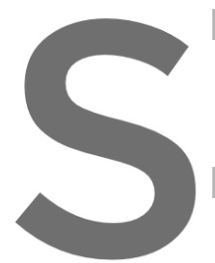

[7] ZANK, M. The Newmark nethod and a space-t
Numerical Mathematics and Advanced Applicatio
Lecture Notes in Computational Science and Eng
[8] ZLoTnIK, A. A. Convergence rate estimates of
equations. In Numerical methods and application
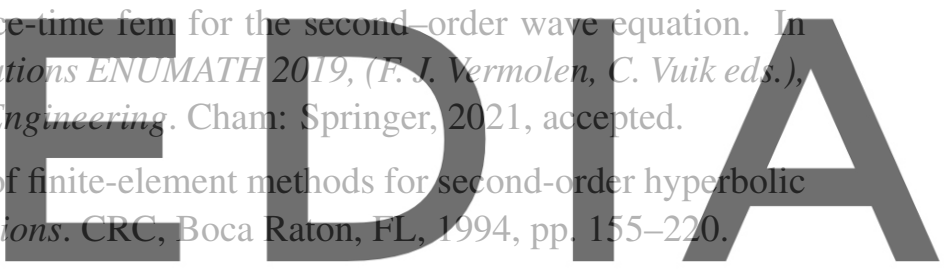

Register for free at https//www.scipedia.com to download the version without the watermark 